\title{
Mitochondrial dynamics regulates hypoxia-induced migration and antineoplastic activity of cisplatin in breast cancer cells
}

\author{
XIAO-JIAN HAN ${ }^{1}$, ZHANG-JIAN YANG ${ }^{1}$, LI-PING JIANG ${ }^{3}$, YONG-FANG WEI ${ }^{1}$, MING-FANG LIAO ${ }^{1,3}$, \\ YISONG QIAN $^{1}$, YONG LI ${ }^{1}$, XUAN HUANG $^{1}$, JIAN-BIN WANG ${ }^{1}$, HONG-BO XIN $^{1}$ and YU-YING WAN ${ }^{1,2}$ \\ ${ }^{1}$ Institute of Translational Medicine, Nanchang University; ${ }^{2}$ Department of Hospital Infection, \\ The Second Affiliated Hospital of Nanchang University; ${ }^{3}$ Department of Pharmacology, \\ School of Pharmaceutical Science, Nanchang University, Nanchang, P.R. China
}

Received October 2, 2014; Accepted November 19, 2014

DOI: 10.3892/ijo.2014.2781

\begin{abstract}
Mitochondria are high dynamic organelles with frequent fission and fusion. Here, we found hypoxia stimulated Drpl expression, mitochondrial fission and migration in metastatic MDA-MB-231 cells, but not in non-metastatic MCF-7 cells. Inhibition of Drp1-dependent mitochondrial fission by Mdivi-1 or silencing Drp1 attenuated hypoxia-induced mitochondrial fission and migration in MDA-MB-231 cells. On the other hand, cisplatin induced significant apoptosis and mitochondrial fission in MDA-MB-231 cells, but not in MCF-7 cells. Mdivi-1 and silencing Drp1 also efficiently prevented cisplatin-induced MMP decrease, ROS production and apoptosis in MDA-MB-231 cells. Our data suggest that Drp1-dependent mitochondrial fission not only regulates hypoxia-induced migration of breast cancer cells, but also facilitates its sensitivity to chemotherapeutic agents. Thus, targeting Drp1-dependent mitochondrial dynamics may provide a novel strategy to suppress breast cancer metastasis and improve the chemotherapeutic effect in the future.
\end{abstract}

\section{Introduction}

Breast cancer is the most common malignant tumor and the leading cause of cancer death in females worldwide (1). Metastasis to vital organs such as lung, liver, bone and brain is responsible for the majority of breast cancer deaths (2). The migration and invasion are the two main aspects of metastatic activity. For metastasis, cancer cells need to migrate and invade into lymphatic or vascular system, and be colonized the metastatic site $(3,4)$. Increasing evidence suggests that

Correspondence to: Dr Xiao-Jian Han or Dr Yu-Ying Wan, Institute of Translational Medicine, Nanchang University, 1299 Xuefu Road, Honggu District, Nanchang, Jiangxi 330031, P.R. China

E-mail: hanxiaojian@hotmail.com

E-mail:wanyy79@hotmail.com

Key words: hypoxia, Drp1, cell migration, cisplatin resistance, breast cancer the metastatic activity is mainly activated by two factors: the intrinsic genetic properties of cancer cells and the tumor microenvironment $(5,6)$. Hypoxia is the common characteristic of solid tumor microenvironment, and also the major stimulator of migration and invasion $(7,8)$. It is well documented that hypoxia modifies cellular activities via stabilizing HIF-1 $\alpha$. As a transcription factor, HIF-1 $\alpha$ promotes the adaption of tumor cells to hypoxia through upregulating gene expression related to cell mobility, angiogenesis, and glycolysis such as MMPs, VEGF and GLUT1 $(7,9,10)$. Furthermore, HIF-1 $\alpha$ overexpression is more frequently observed in the metastases than in the primary tumor of breast cancer, and is correlated with distant metastasis and poor prognosis (11). Therefore, hypoxia plays an important role in the metastasis and poor prognosis of breast cancer.

Mitochondria are vital organelles for ATP production and intracellular $\mathrm{Ca}^{2+}$ homeostasis. As such, they are involved in a variety of cellular processes, including differentiation, proliferation and apoptosis (12-14). Furthermore, mitochondria are highly dynamic organelles with frequent fission and fusion events, and move through the cells (15). Mitochondrial dynamics is important to maintain the normal shape, structure, quantity and function of mitochondria and can respond to a variety of extrinsic environments (16). It has been well-recognized that the abnormal mitochondrial dynamics potentially contributes to tumorigenesis (17). On the other hand, some highly conserved dynamin-related GTPases are identified as the mediator of mitochondrial dynamics. The process of mitochondrial outer membrane fission is mediated by dynamin-related protein 1 (Drp1) and Fis1 in mammalian cells. In contrast, OPA-1 and Mitofusins (Mfn1 and Mfn2) are required for the fusion of mitochondrial inner and outer membrane, respectively $(16,17)$. In a recent study $(18)$, mitochondrial fission was found to regulate the migration and invasion of breast cancer cells. The Drp1 expression in metastatic MDA-MB-231 cells is higher than that in non-metastatic MCF-7 cells. Drp1-dependent mitochondrial fission redistributes mitochondria in lamellipodial regions and enhances the migratory activity of breast cancer cells through promotion of lamellipodia formation. However, the role of Drp1 in migration of breast cancer cells is only investigated under normoxia, and the different migratory activity between metastatic and 
non-metastatic breast cancer cells is mainly determined by the intrinsic genetic properties of two cell lines. As mentioned above, metastatic activity can also be stimulated by the local characteristics of tumor microenvironment such as hypoxia. Thus, it is necessary to further investigate the role of mitochondrial dynamics in hypoxia-induced migration of breast cancer cells.

Platinum-based drugs are widely used in the treatment of cancer such as lymphomas, melanoma, head-neck cancer, bladder cancer and gynaecological tumors (19). Cisplatin is the first platinum-based drug, discovered in the 1960s (20). Cisplatin interacts with DNA double strands by formation of interstrand and intrastrand adducts, thereby induces apoptosis in cancer cells through the interference with DNA replication and gene transcription (21). Similar to other chemotherapeutic agents, the effect of cisplatin is commonly limited by the resistance of cancer cells. Cisplatin resistance can be intrinsic or acquired. Intrinsic resistance means that cancer cells retain certain featured gene expression profile contributing to resistance prior to cisplatin treatment. In contrast, the acquired resistance occurs in cancer cells after cisplatin-induced epigenetic modulation and gene mutation (21). Interestingly, recent studies suggest the possible role of mitochondrial dynamics in the acquired cisplatin resistance or sensitivity $(22,23)$. OPA-1mediated mitochondrial fusion is potentially responsible for cisplatin-induced resistance in neuroblastoma B50 rat cells (22). By contrast, Drp1-dependent mitochondrial fission was found to regulate piceatannol-induced cisplatin sensitivity in ovarian cancer (23). Moreover, it was reported that nonmetastatic MCF-7 cells were more resistant to cisplatin than metastatic MDA-MB-231 cells (24), and Drp1 expression level in MCF-7 cells is also lower than that in MDA-MB-231 cells (18). Thus, it is of interest to investigate whether intrinsic Drp1-dependent mitochondrial dynamics regulates cisplatin resistance in breast cancer cells.

In the present study, we found hypoxia upregulated Drp1 expression and stimulated mitochondrial fission in metastatic breast cancer MDA-MB-231, but not in non-metastatic MCF-7 cells. The hypoxia-induced migration in MDA-MB-231 was also stronger than that in MCF-7 cells. Inhibition of Drp1dependent mitochondrial fission by Mdivi-1 or silencing Drp1 significantly attenuated hypoxia-induced mitochondrial fission and migration in MDA-MB-231 cells. On the other hand, CDDP treatment stimulated mitochondrial fission and induced significant apoptosis in MDA-MB-231 cells, but not in MCF-7 cells. Similarly, inhibition of Drp1-dependent mitochondrial fission by Mdivi-1 or silencing Drp1 effectively prevented CDDP-induced MMP decrease, ROS production and apoptosis in MDA-MB-231 cells. These results indicate the role of Drp1-dependent mitochondrial dynamics in hypoxia-induced migration and antineoplastic activity of cisplatin in breast cancer cells.

\section{Materials and methods}

Cell culture. Human breast cancer MDA-MB-231 and MCF-7 cell lines were obtained from the American Type Culture Collection (ATCC). MDA-MB-231 cells were grown in RPMI-1640 media (HyClone, South Logan, UT, USA) supplemented with $10 \%$ fetal bovine serum (Trans Serum ${ }^{\mathrm{TM}}$, Beijing,
China) and $1 \%$ penicillin and streptomycin (P/S) (Solarbio, Beijing, China). MCF-7 cells were grown in Dulbecco's modified Eagle's medium (HyClone) with $10 \%$ fetal bovine serum and $1 \% \mathrm{P} / \mathrm{S}$. For normoxic culture, the cells were maintained in a humidified incubator at $37^{\circ} \mathrm{C}$ with an atmosphere containing $5 \% \mathrm{CO}_{2}$. For hypoxia treatment, the cells were transferred to a humidified hypoxic incubator (Thermo Scientific, MA, USA) containing $1 \% \mathrm{O}_{2}, 5 \% \mathrm{CO}_{2}$ and $94 \% \mathrm{~N}_{2}$ at $37^{\circ} \mathrm{C}$.

Wound healing and transwell assay. The wound healing and transwell assays were carried out as previously described (25). For wound healing assay, MDA-MB-231 and MCF-7 cells were firstly seeded on 35-mm dishes and maintained in growth medium. Briefly, a scratch with constant width was done in monolayer of cells with a $200-\mu l$ pipette tip. The cells were washed twice with PBS to remove the suspended cells and further cultured in medium without supplement of fetal bovine serum under normoxia or hypoxia. To inhibit Drp1-dependent mitochondrial fission, Drp1 inhibitor Mdivi-1 (Sigma-Aldrich, St. Louis, MO, USA) at $5 \mu \mathrm{M}$ and silencing Drp1 with siRNA (Biotend, Shanghai, China) were introduced to cells. Wound closure was photographed at different time-points after scratch by bright-field microscopy (Olympus, Tokyo, Japan).

Transwell assay was performed with transwell chamber (Corning, Inc., NY, USA). In brief, 1.0×104 MDA-MB-231 cells or $1.4 \times 10^{4} \mathrm{MCF}-7$ cells were seeded into the upper chamber with $200 \mu \mathrm{l}$ of serum-free medium. The upper chamber was incubated in $500 \mu \mathrm{l}$ of complete medium containing $10 \%$ fetal bovine serum and $1 \% \mathrm{P} / \mathrm{S}$. After normoxic or hypoxic incubation (7-h incubation for MDA-MB-231 and 18-h incubation for MCF-7 cells, respectively), the cells on the top surface of the insert were gently removed with a cotton swab. The migrated cells on lower surface were fixed with $4 \%$ paraformaldehyde (Sigma-Aldrich) and stained with crystal violet (Sigma-Aldrich) for $30 \mathrm{~min}$. The migrated cells were further photographed and counted in four random fields. All assays were independently repeated at least in triplicate.

Western blot analysis. MDA-MB-231 and MCF-7 cells were harvested and lyzed by radioimmunoprecipitation assay (RIPA) lysis buffer (Solarbio) according to the manufacturer's instructions. The whole cell lysates were mixed with equal volume of $2 \mathrm{X}$ loading buffer (25\% glycerol, $2 \%$ sodium dodecyl sulfate, $5 \% \beta$-mercaptoethanol, $0.01 \%$ bromophenol blue, and $1 \mathrm{M}$ Tris- $\mathrm{HCl}$ ), sonicated, boiled for $5 \mathrm{~min}$ and stored at $-20^{\circ} \mathrm{C}$ prior to use. The cell lysates were subjected to SDS-PAGE gel electrophoresis. After electrophoresis, the proteins were transferred onto PVDF membrane (Millipore, MA, USA). The membrane was blocked with 5\% skim milk in TBST buffer for $1 \mathrm{~h}$ at room temperature, and then immunoblotted for $2 \mathrm{~h}$ at room temperature with the following primary antibody: rabbit anti-Drp1 and Mfn2 antibody (Cell Signaling, Boston, MA, USA, 1:1,000), rabbit anti-Mfn1 and OPA-1 antibody (Abcam, Cambridge, UK, 1:1,000), and rabbit anti-GAPDH antibody (Santa Cruz Biotechnology, TX, USA, 1:1,000). After three washes with TBST, the membranes were further incubated with an HRP-conjugated goat anti-rabbit secondary antibody (TransGen Biotech, Beijing, China, 1:2,000) for $2 \mathrm{~h}$ at room temperature. Chemiluminescence assay was carried out with Amersham ECL Prime Western Blotting Detection reagents 
(CWBIO, Beijing, China), and the immunobloting signal was detected using Molelular Imager ${ }^{\circledR}$ Chemi DOC ${ }^{\mathrm{T}} \mathrm{XRS}^{+}$system (Bio-Rad, CA, USA).

RNA interference. For RNA interference, Drp1 siRNA and scramble siRNA were chemically synthesized (Drp1 siRNAs: 5'-GAGGUUAUUGAACGACUCAdTdT-3' and 5'-TGAGT CGTTCAATAACCTCdTdT-3', scramble siRNAs: 5'-UUCUC CGAACGUGUCACGUdTdT-3' and 5'-ACGUGACACG UUCGGAGAAdTdT-3'), respectively. Annealed siRNAs (30 $\mathrm{nM}$ ) were transfected into MDA-MB-231 cells using Lipofectamine 2000 (Invitrogen, CA, USA) according to the manufacturer's instructions. Drp1 expression was further examined by western blotting to evaluate the silencing efficiency at 24,48 and $72 \mathrm{~h}$ after transfection.

Mitochondrial imaging. As described previously (26), pDsRed2-Mito was transfected into MDA-MB-231 and MCF-7 cells with Lipofectamine 2000 to label mitochondria. Briefly, mitochondrial morphology was observed under normoxia and 8 h-hypoxia. In addition, mitochondrial morphology was also examined after treatment with $30 \mu \mathrm{M}$ of CDDP (Xiya Reagent, Sichuan, China) for $8 \mathrm{~h}$. To examine the role of Drp1 in hypoxia or CDDP-induce mitochondrial dynamics, cells were transfected with Drpl siRNAs $24 \mathrm{~h}$ prior to stimulation or pretreated with $5 \mu \mathrm{M}$ Mdivi-12 h prior to stimulation. After the indicated treatments, cells were fixed with $4 \%$ PFA, and mitochondrial morphology was observed under an inverted fluorescence microscope (Olympus, Tokyo, Japan) with excitation at $545 \mathrm{~nm}$.

Detection of the intracellular ROS level. MDA-MB-231 cells were cultured to be $90 \%$ confluent at the time of analysis. To examine the role of Drp1-dependent mitochondrial fission in intracellular ROS production, cells were pretreated with $5 \mu \mathrm{M}$ Mdivi-1 for $2 \mathrm{~h}$ or transfected with Drp1 siRNAs $24 \mathrm{~h}$ prior to $30 \mu \mathrm{M}$ CDDP treatment. To detect the intracellular ROS level, cells were incubated with $10 \mu \mathrm{M}$ of the fluorescent probe 2',7'-dichlorodihydrofluorescein diacetate (DCFH-DA, Sigma-Aldrich) for $30 \mathrm{~min}$ at $37^{\circ} \mathrm{C}$ in the dark. After incubation, the cells were washed twice with PBS and harvested. The fluorescence intensity was measured using flow cytometry (Guava, Millipore Corp.) with the excitation source at $488 \mathrm{~nm}$ and emission wavelength of $525 \mathrm{~nm}$. Data analysis was carried out using inCyte software (Guava, Millipore Corp.).

Measurement of mitochondrial membrane potential $(\Delta \psi m)$. The mitochondrial membrane potential $(\Delta \psi \mathrm{m})$ of MDA-MB-231 cells were measured by flow cytometry using tetramethylrhodamine ethyl ester (TMRE, Invitrogen), a potentiometric, cell-permeable fluorescent indicator that accumulates in the highly negatively charged interior of mitochondria. The cells were incubated with $50 \mathrm{nM}$ of TMRE for $20 \mathrm{~min}$ at $37^{\circ} \mathrm{C}$. After incubation, the cells were washed twice with PBS and harvested for the analysis by flow cytometry with the excitation and emission wavelength at 540 and $575 \mathrm{~nm}$, respectively.

Annexin V-FITC/PI apoptosis assay. After the indicated treatments, MDA-MB-231 cells were harvested from each group for apoptosis assay using Annexin V-fluorescein isothiocyanate (Annexin V-FITC) and propidium iodide (PI) (4Abio, Beijing, China) double staining. The cells were resuspended in $100 \mu \mathrm{l}$ binding buffer with $5 \mu \mathrm{l}$ Annexin V-FITC and $200 \mathrm{ng}$ PI and incubated for $15 \mathrm{~min}$ at room temperature in the dark. Then, the samples were subjected to apoptosis assay and cytometry, and the data were processed using Guawa Nexin software (Guava, Millipore Corp.).

Statistical analysis. The quantitative data are shown as the mean \pm SD. Data were analyzed using either Student's t-test to compare two conditions or ANOVA followed by planned comparisons of multiple conditions, and $\mathrm{p}<0.05$ was considered to be significant.

\section{Results}

The response of MDA-MB-231 and MCF-7 cells to hypoxia is different in mitochondrial dynamics andmigration. In the most recent study (18), it was found that mitochondrial dynamics regulated the migration of breast cancer cells under normoxia. Drp1 expression level in metastatic MDA-MB-231 cells was higher than that in non-metastatic MCF-7 cells. Mitochondrial fission regulated the migration and invasion of breast cancer cells through modifying lamellipodial formation. However, it is still unknown whether mitochondrial dynamics also contributes to the response of breast cancer cells to hypoxia. In the present study, we confirm the migratory activity, Drp1 expression and mitochondrial morphology in MDA-MB-231 and MCF-7 cells. Compared to non-metastatic MCF-7 cells, metastatic MDA-MB-231 cells retained stronger migration, higher Drp1 expression and more fragmented mitochondria (Fig. 1A-C). These results are consistent with a previous study (18). To investigate the possible role of hypoxia in mitochondrial dynamics in two cell lines, the expression level of mitochondria-related proteins after hypoxia was examined by western blot assay. As shown in Fig. 1D, Drp1 expression was significantly upregulated from 6 to $24 \mathrm{~h}$ post-hypoxia in MDA-MB-231 cells, but not in MCF-7 cells. However, the expression of Mfn1, Mfn2 and OPA-1 in two cell lines was not obviously altered up to $24 \mathrm{~h}$ after hypoxia. Based on the alteration in mitochondria-related protein expression, mitochondrial morphology after hypoxia in two cell lines was further examined. Hypoxia induced mitochondrial fission in MDA-MB-231 cells, but not in MCF-7 cells (Fig. 2A). In addition, the response of two breast cancer cell lines to hypoxia was also different in migration. Both wound healing and transwell assay showed that hypoxia significantly increased the migratory activity in MDA-MB-231 cells, but not in MCF-7 cells (Fig. 2B-F). These results suggest the possibility that Drp1-dependent mitochondrial fission might contribute to the different response of two breast cancer cell lines to hypoxia.

Inhibition of Drpl-dependent mitochondrial fission efficiently attenuates hypoxia-induced migration of MDA-MB-231 cells. In our previous study, we found that hypoxia upregulated the expression of Drp1 and stimulated mitochondrial fission in glioblastoma U251 cells. Drp1-dependent mitochondrial fission was involved in hypoxia-induced migration in U251 cells (25). In the present study, non-metastatic MCF-7 and 


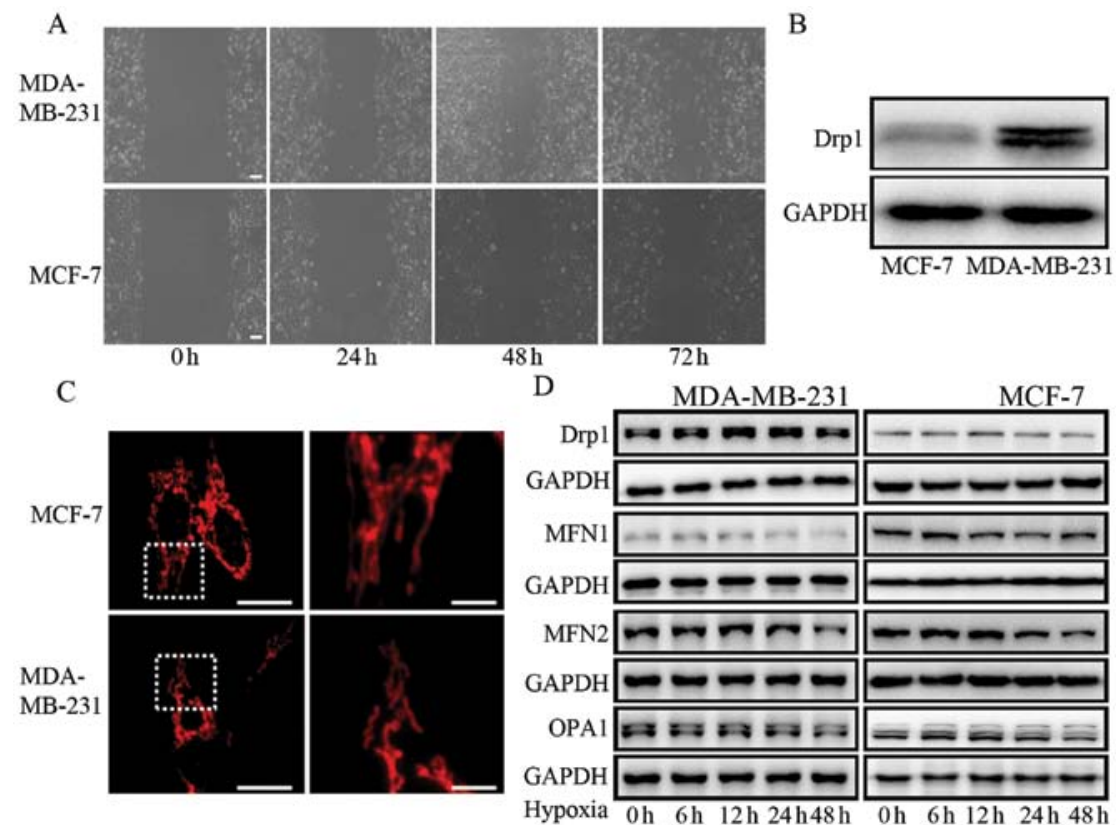

Figure 1. The different migratory activity, mitochondrial morphology and response to hypoxia between MDA-MB-231 and MCF-7 cells. (A) The migratory activity of MDA-MB-231 and MCF-7 cells under normoxia. The cells were scraped with a 200- $\mu$ l pipette tip and cultured under normoxia. Cell migration was examined by wound healing assay at the indicated time-points. Scale bar, $100 \mu \mathrm{m}$. (B) The Drp1 expression level in MDA-MB-231 and MCF-7 cells under normoxia. GAPDH was used as endogenous reference. (C) The mitochondrial morphology in MDA-MB-231 and MCF-7 cells under normoxia. MDA-MB-231 and MCF-7 cells were transfected with pDsRed2-Mito to label mitochondria. Long scale bar, $20 \mu \mathrm{m}$; short scale bar, $5 \mu \mathrm{m}$. (D) Effect of hypoxia on the expression of GTPases required for mitochondrial dynamics in MDA-MB-231 and MCF-7 cells. Cells were incubated under hypoxia and harvested for immunoblot assay at the indicated time-points. The expression level of Drp1, Mfn1, Mfn2 and OPA-1 after hypoxia was examined. GAPDH was used as endogenous reference.

A

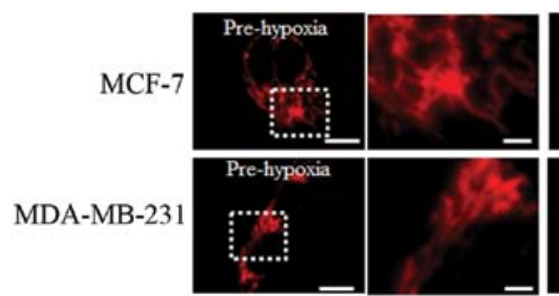

B

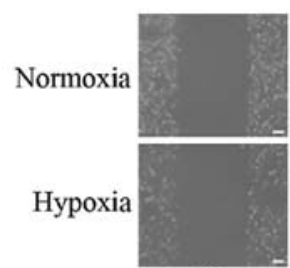

$0 \mathrm{~h}$

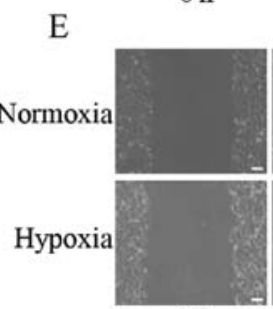

$0 \mathrm{~h}$
MDA-MB-231

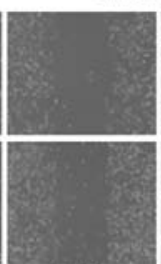

$24 \mathrm{~h}$

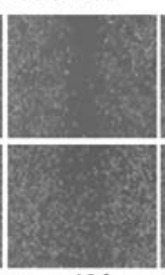

$48 \mathrm{~h}$

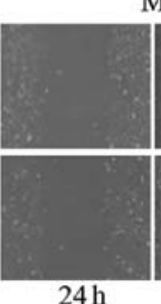

$24 \mathrm{~h}$

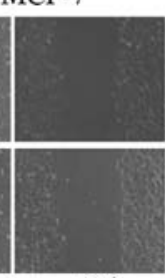

$48 \mathrm{~h}$

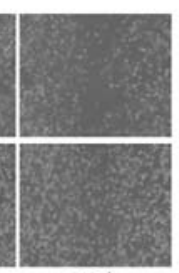

$72 \mathrm{~h}$

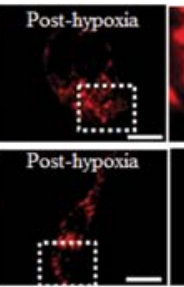

C

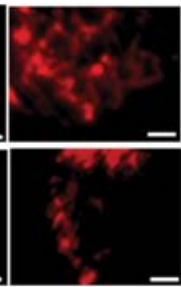

D

20,
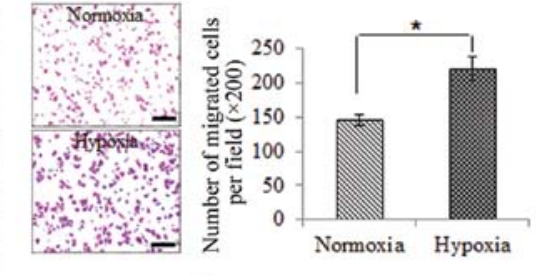

$\mathrm{F}$ G
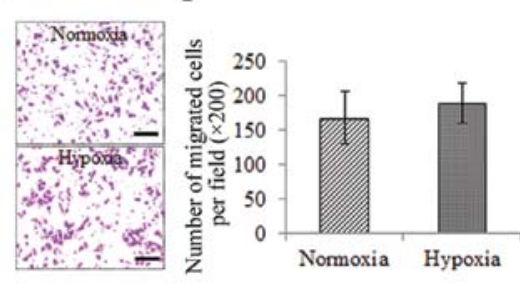

$72 \mathrm{~h}$

Figure 2. Hypoxia-induced mitochondrial dynamics and migration are different between MDA-MB-231 and MCF-7 cells. (A) The mitochondrial morphology in MDA-MB-231 and MCF-7 cells under hypoxia. Mitochondrial morphology in MDA-MB-231 and MCF-7 cells was observed prior to hypoxia or after 8 h-hypoxia incubation using fluorescence microscopy. Long scale bar, $20 \mu \mathrm{m}$; short scale bar, $5 \mu \mathrm{m}$. (B-D) Effect of hypoxia on migration of MDA-MB-231 cells. The cells were cultured under normoxia or hypoxia after scratch, and cell migration was examined by wound healing assay at the indicated time-points. Scale bar, $100 \mu \mathrm{m}$ (B). The migratory activity of MDA-MB-231 cells under normoxia or hypoxia was evaluated by transwell assay. The migrated cells were visualized by microscopy. Scale bar, $100 \mu \mathrm{m}(\mathrm{C})$. In addition, cell migration was quantified by mean cell counts from at least 9 fields in three independent experiments for each condition. * ${ }^{*}<0.05$ (D). (E-G) Effect of hypoxia on migration of MCF-7 cells. The cells were cultured under normoxia or hypoxia after scratch, and cell migration was examined by wound healing assay at the indicated time-points. Scale bar, $100 \mu \mathrm{m}$ (E). The migratory activity of MCF-7 cells under normoxia or hypoxia was evaluated by transwell assay. The migrated cells were visualized by microscopy. Scale bar, $100 \mu \mathrm{m}$ (F). Cell migration was also quantified by mean cell counts from at least 9 fields in three independent experiments for each condition (G). 


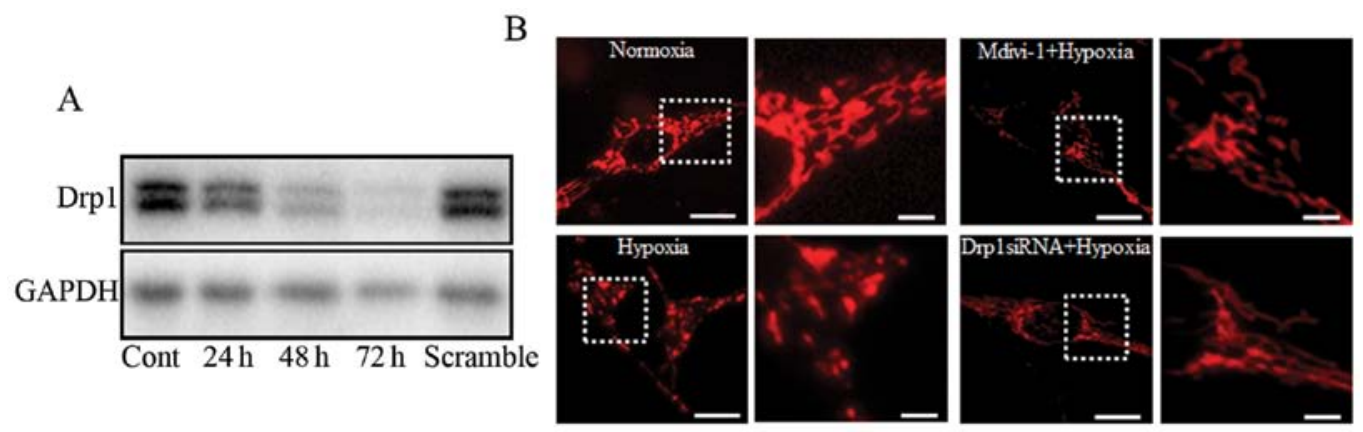

$\mathrm{C}$

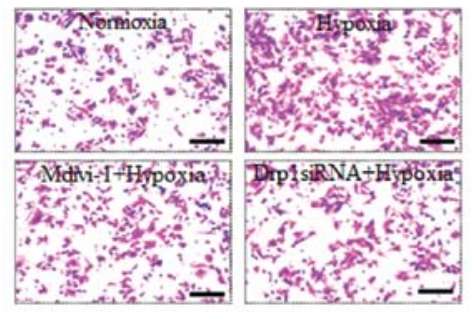

D

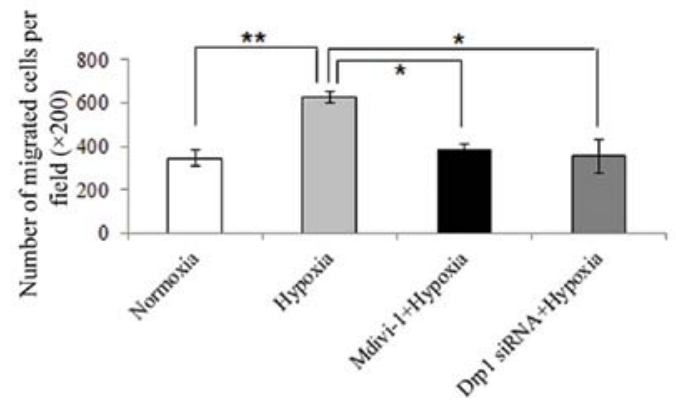

Figure 3. Inhibition of Drp1-dependent mitochondrial fission efficiently attenuates hypoxia-induced migration of MDA-MB-231 cells. (A) Silencing Drp1 in MDA-MB-231 cells by siRNA. The cells were transfected with siRNA targeted to Drp1 or scramble siRNA. Whole cell lysate prepared at the indicated time-points after transfection were subjected to immunoblotting for Drp1. GAPDH was used as endogenous reference. (B) Effect of Mdivi-1 and silencing Drpl on hypoxia-induced mitochondrial fission in MDA-MB-231 cells. The cells were fixed with $4 \%$ PFA after normoxic or hypoxic incubation for 8 h, and mitochondrial morphology was observed by fluorescence microscopy. To inhibit Drp1-dependent mitochondrial fission, the cells were pretreated with $5 \mu \mathrm{M}$ Mdivi-1 or transfected with Drp1 siRNA prior to hypoxia. Long scale bar, $20 \mu \mathrm{m}$; short scale bar, $5 \mu \mathrm{m}$. (C and D) Drp1-dependent mitochondrial fission on hypoxia-induced migration of MDA-MB-231 cells. The migratory activity of MDA-MB-231 cells under normoxia or hypoxia was evaluated by transwell assay. The migrated cells in each condition were visualized by microscopy. Scale bar, $100 \mu \mathrm{m}(\mathrm{C})$. In addition, cell migration was quantified by mean cell counts from at least 9 fields in three independent experiments for each condition. ${ }^{*} \mathrm{p}<0.05,{ }^{* *} \mathrm{p}<0.01$ (D).

metastatic MDA-MB-231 cells differently responded to hypoxia in Drp1 expression and migration. To investigate the role of Drp1 in hypoxia-induced migration of MDA-MB-231 cells, Drp1 inhibitor Mdivi-1 and silencing Drp1 by siRNA were used to inhibit Drp1-dependent mitochondrial dynamics. First, the specific knockdown of Drp1 in MDA-MB-231 cells by siRNA was examined by western blot assay. As shown in Fig. 3A, Drp1 expression was significantly decreased at 48 and $72 \mathrm{~h}$ after transfection of Drp1 siRNA. By contrast, no significant effect of scramble siRNA on Drp1 expression was detected. Importantly, pre-treatment with $5 \mu \mathrm{M}$ of Mdivi-1 and silencing Drp1 not only efficiently attenuated hypoxia-induced mitochondrial fission in MDA-MB-231 cells (Fig. 3B), but also significantly prevented hypoxia-induced migration in MDA-MB-231 cells (Fig. 3C and D). Together, these results indicate that Drp1-dependent mitochondrial fission regulates hypoxia-induced migration in metastatic MDA-MB-231 cells.

Effect of $C D D P$ on cell survival and mitochondrial dynamics in MDA-MB-231 and MCF-7 cells. CDDP is widely used in the treatment of cancer. However, the sensitivity to CDDP varies in different cancer cells (19). In breast cancer cells, it has been reported that non-metastatic MCF-7 cells are more resistant to CDDP than metastatic MDA-MB-231 cells (24). In the present study, we found that $30 \mu \mathrm{M}$ of CDDP induced apoptosis in MDA-MB-231 and MCF-7 cells at 18.5 and $13.2 \%$, respectively (Fig. 4A-D). The result of statistical analysis showed that $30 \mu \mathrm{M}$ of CDDP induced significant apoptosis in MDA-MB-231 cells, but not in MCF-7 cells (Fig. 4B and D). These results are consistent with a previous study (24). In addition, recent studies suggest that mitochondrial dynamics possibly contributes to the CDDP resistance or sensitivity $(22,23)$. Thereby, we further examined the alteration in mitochondrial morphology after CDDP in two cell lines. As shown in Fig. 4E, $30 \mu \mathrm{M}$ of CDDP stimulated mitochondrial fission in MDA-MB-231 cells, but not in MCF-7 cells. These results indicate that CDDP has different effect on cell survival and mitochondrial dynamics in the two breast cancer cell lines.

Inhibition of Drpl-dependent mitochondrial fission desensitizes $M D A-M B-231$ cells to $C D D P$. Mitochondria are important organelles for cell survival through ATP production and regulation of intracellular $\mathrm{Ca}^{2+}$. In addition, mitochondria are also highly dynamic with frequent fission and fusion (27). Emerging evidence indicates that mitochondrial dynamics is involved in a variety of cellular processes, including proliferation, differentiation and apoptosis (12-14). To investigate the role of mitochondrial dynamics in antineoplastic activity of CDDP in MDA-MB-231 cells, Mdivi-1 and silencing Drp1 were used to inhibit Drp1-dependent mitochondrial fission. Apoptosis assay was carried out by flow cytometry using Annexin V-FITC/PI double staining. As shown in Fig. 5A, $5 \mu \mathrm{M}$ of Mdivi-1 and silencing Drp1 efficiently attenuated 
A

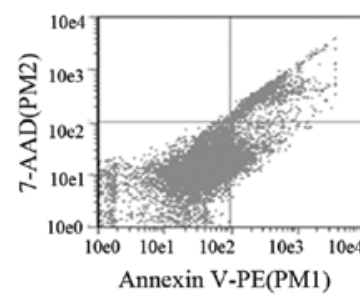

$\mathrm{C}$

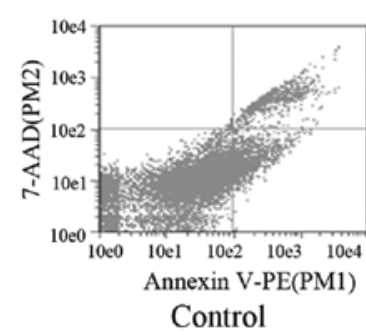

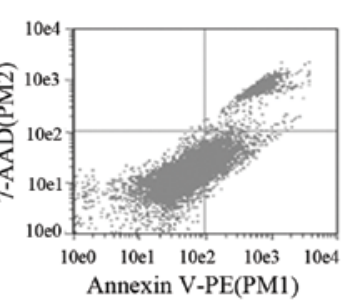

CDDP

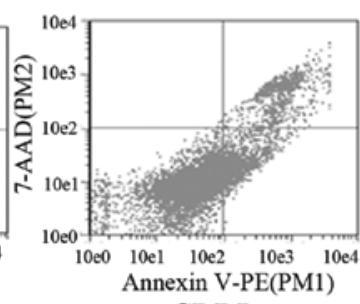

CDDP
B

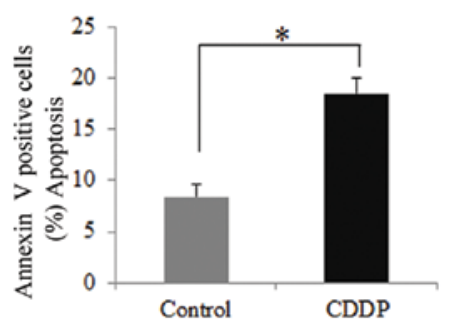

$\mathrm{D}$

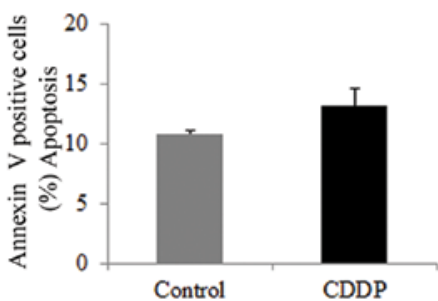

$\mathrm{E}$
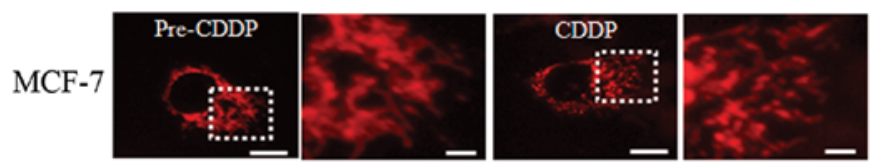

MDA-MB-231
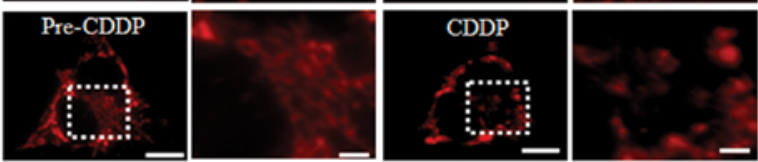

Figure 4. Effect of CDDP on cell survival and mitochondrial morphology in MDA-MB-231 and MCF-7 cells. (A-D) Effect of CDDP on cell survival in MDA-MB-231 and MCF-7 cells. After treatment with $30 \mu \mathrm{M}$ of CDDP, the apoptosis of two cell lines was examined by flow cytometry using Annexin V-FITC/PI double staining. (A and B) Results of MDA-MB-231 cells. "p<0.05. (C and D) Results of MCF-7 cells. (E) Mitochondrial morphology in MDA-MB-231 and MCF-7 cells after CDDP. Mitochondrial morphology in MDA-MB-231 and MCF-7 cells was observed prior to CDDP or at $8 \mathrm{~h}$ post CDDP using fluorescence microscopy. Long scale bar, $20 \mu \mathrm{m}$; short scale bar, $5 \mu \mathrm{m}$.

A
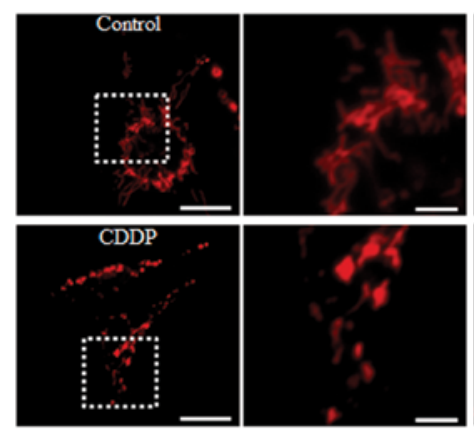

B
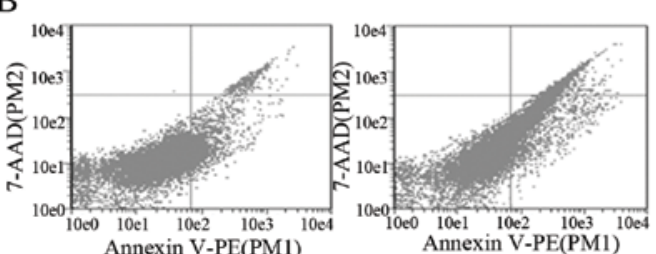

Contro
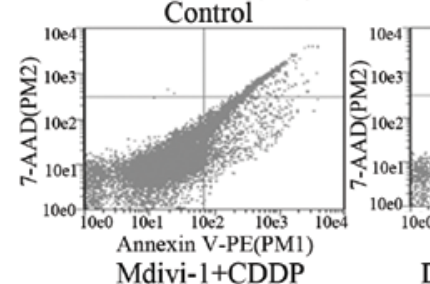
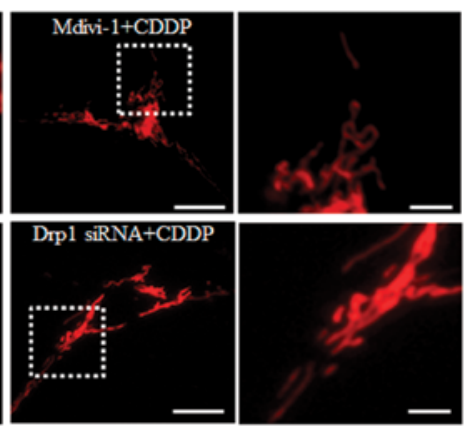

$\mathrm{C}$

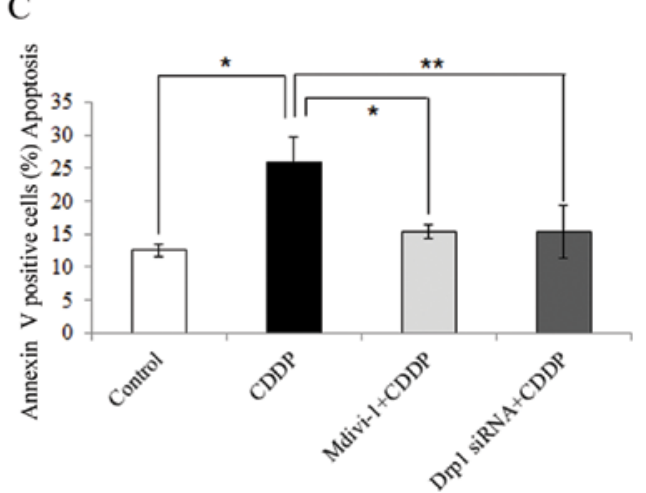

Figure 5. Drp1-dependent mitochondrial fission on the antineoplastic activity of CDDP in MDA-MB-231 cells. (A) Mdivi-1 and silencing Drp1 efficiently attenuated CDDP-induced mitochondrial fission. MDA-MB-231 cells were fixed with 4\% PFA in each condition, and mitochondrial morphology was observed by fluorescence microscopy. Long scale bar, $20 \mu \mathrm{m}$; short scale bar, $5 \mu \mathrm{m}$. (B) Drp1-dependent mitochondrial fission on CDDP-induced apoptosis in MDA-MB-231 cells. After the indicated treatments, the apoptosis of MDA-MB-231 cells was examined by flow cytometry using Annexin V-FITC/PI double staining. (C) The quantification of apoptosis in each group was performed. The data present the mean of three independent experiments. ${ }^{*}$ p $<0.05,{ }^{* *}$ p $<0.01$. 


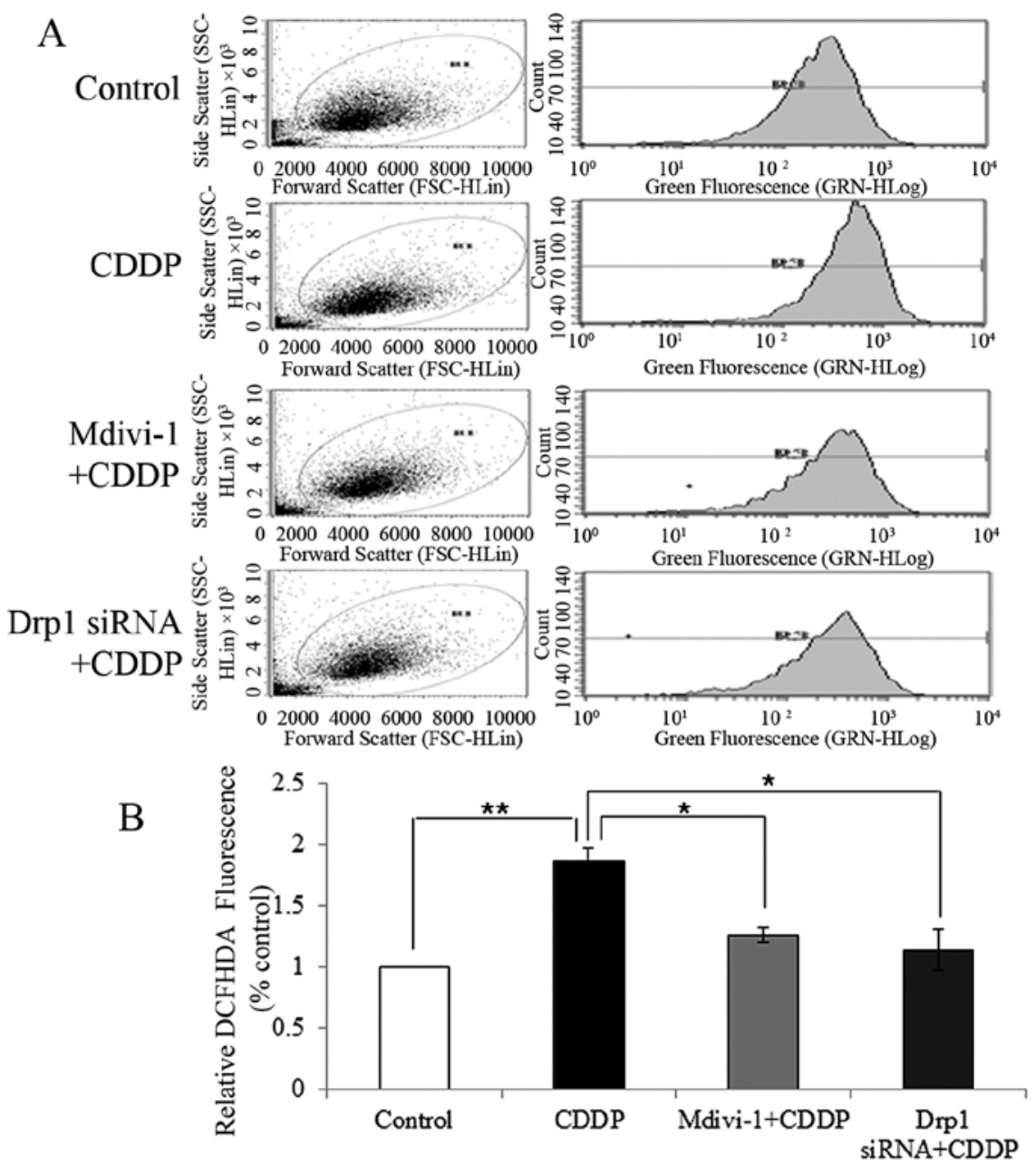

Figure 6. Drp1-dependent mitochondrial fission on the intracellular ROS level in MDA-MB-231 cells after CDDP. (A) After the indicated treatments, the intracellular ROS level in MDA-MB-231 cells was examined by flow cytometry using DCFH-DA staining. (B) The relative ROS level was quantified in each group. The data present the mean of at least three independent experiments. ${ }^{*} \mathrm{p}<0.05,{ }^{* *} \mathrm{p}<0.01$.

CDDP-induced mitochondrial fission in MDA-MB-231 cells. Moreover, inhibition of Drp1-dependent mitochondrial fission by Mdivi-1 and silencing Drp1 significantly prevented CDDP-induced apoptosis in MDA-MB-231 cells (Fig. 5B and $\mathrm{C}$ ). These results suggest that Drp1-dependent mitochondrial dynamics contributes to the sensitivity of metastatic MDA-MB-231 cells to CDDP.

Drpl-dependent mitochondrial fission on CDDP-induced ROS production and MMP decrease in MDA-MB-231 cells. It was reported that CDDP induced the disruption of mitochondrial dynamics in cancer cells (22). Moreover, mitochondrial dynamics is relevant to the maintenance of intracellular ROS and MMP (28). Disruption of mitochondrial dynamics disturbs mitochondrial functions, thereby influence intracellular ROS production and MMP $(28,29)$. To investigate the mechanism of mitochondrial dynamics in the antineoplastic activity of CDDP in MDA-MB-231 cells, the intracellular ROS level and MMP were measured. As shown in Fig. 6, CDDP treatment increased the intracellular ROS to 1.86-fold of control. In contrast, pre-treatment with Mdivi-1 and silencing Drp1 significantly inhibited CDDP-induced intracellular ROS increment. In addition, CDDP significantly decreased the
MMP to $76.1 \%$ of control. Pre-treatment with Mdivi-1 and silencing Drp1 partially recovered the MMP after CDDP to 84.7 and $88.3 \%$ of control (Fig. 7). Taken together, it suggests that Drp1-dependent mitochondrial dynamics may regulate the antineoplastic activity of CDDP in MDA-MB-231 cells through the impact on intracellular ROS and MMP.

\section{Discussion}

Cancer cells with dis-regulated cell cycle and uncontrolled high proliferation frequently lead to a characteristic hypoxia microenvironment in the central region of solid tumor. Hypoxia is an important factor stimulating the migration and invasion of cancer cells $(7,8,30)$. On the other hand, mitochondria play vital roles in cancer cell survival, proliferation and metastasis though regulation of energy metabolism, $\mathrm{Ca}^{2+}$ homeostasis, cell cycle and apoptosis (12-14). It has been well-recognized that mitochondrial dysfunction is involved in cancer etiology (31-34). Furthermore, mitochondria are high dynamic organelles with frequent fission and fusion events (15). The dynamic balance between fission and fusion is important for maintenance of normal mitochondrial architecture and function (16). Some highly conserved GTPases are identified as the media- 


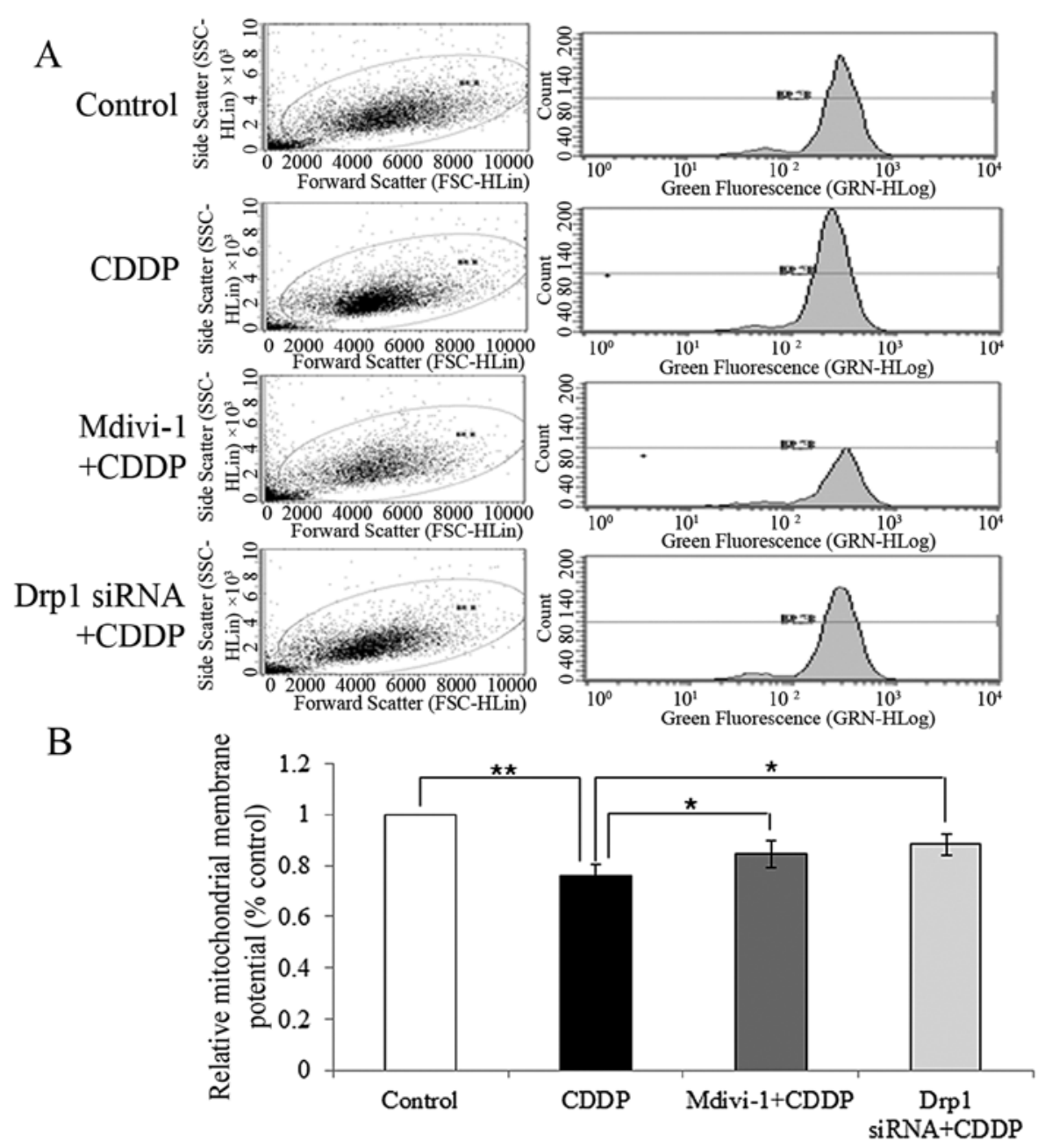

Figure 7. Drp1-dependent mitochondrial fission on CDDP-induced MMP decrease in MDA-MB-231 cells. (A) After the indicated treatments, the MMP in MDA-MB-231 cells was examined by flow cytometry using TMRE staining. (B) The relative MMP was quantified in each group. The data present the mean of at least three independent experiments. ${ }^{*} \mathrm{p}<0.05,{ }^{* *} \mathrm{p}<0.01$.

tors of mitochondrial fission and fusion events $(16,17)$. Drp1 regulates mitochondrial outer membrane fission in collaboration with Fis1. In contrast, Mitofusins (Mfn1 and Mfn2) and OPA-1 are required for the fusion of mitochondrial outer and inner membrane, respectively. Recent studies have shed light on the role of Drp1-dependent mitochondrial fission in cancer biology $(17,18,35,36)$. The high expression of Drp1 is found in cancers such as neuroblastoma, breast cancer and lung cancer $(18,35,36)$. Inhibition of Drp1-dependent mitochondrial fission is an effective means to reduce cancer cell growth and enhance spontaneous apoptosis (37). In our recent study (25), we found that hypoxia could promote the transcription and expression of Drp1, and Drpl-dependent mitochondrial fission is involved in hypoxia-induced migration of U251 cells. In addition, another recent study showed Drp1 expression in metastatic MDA-MB-231 cells is higher than that in non-metastatic MCF-7 cells. Under normoxia, Drp1-dependent mitochondrial fission regulates the migration and invasion of breast cancer cells through redistribution of mitochondria and modification of lamellipodia formation (18). However, the different migratory activity between metastatic and non-metastatic breast cancer cell lines is mainly determined by the intrinsic genetic properties. In fact, migratory activity of cancer cells can be stimulated by the extrinsic environment stress such as hypoxia. Similarly, mitochondria can also respond to the environmental stresses, extracellular stimulations and chemotherapy $(15,22,25)$. Thus, it is necessary to investigate the migration of non-metastatic and metastatic breast cancer cell lines under hypoxia, and explored the role of Drp1-dependent mitochondrial fission in hypoxia-induced migration of human breast cancer cells.

In the present study, we examined the migratory activity, Drp1 expression level and mitochondrial morphology in MCF-7 and MDA-MB-231 cells under normoxia. Consistent with the previous studies (18), stronger migration, higher Drp1 expression level and more fragmented mitochondria were observed in metastatic MDA-MB-231 than that in non-metastatic MCF-7 cells (Fig. 1A-C). To investigate the response of two breast cancer cell lines to hypoxia, we examined the expression level of GTPases required for mitochondrial fission or fusion (Fig. 1D). The expression of Drp1 was increased in MDA-MB-231 cells after hypoxia incubation, especially at 12 and $24 \mathrm{~h}$ post-hypoxia. However, this phenomenon was not observed in MCF-7 cells. Furthermore, hypoxia did not significantly affect the expression of mitochondrial fusion protein OPA-1 and Mfn1 in two breast cancer cell lines. Instead, a 
slight decrease in Mfn2 was detected in both cell lines, especially at $48 \mathrm{~h}$ post-hypoxia. Notably, hypoxia stimulated mitochondrial fission and migratory activity in MDA-MB-231 cells, but not in MCF-7 (Fig. 2). To examine the possible role of Drp1-dependent mitochondrial fission in hypoxiainduced migration, MDA-MB-231 cells were pre-treated with Mdivi-1, a Drp1 inhibitor. Mdivi-1 (5 $\mu \mathrm{M})$ efficiently attenuated hypoxia-induced mitochondrial fission and migration of MDA-MB-231 cells (Fig. 3B-D). However, the Drp1 inhibitor Mdivi-1 could have some off-target effects such as Drp1-independent effect on mitochondrial outer membrane permeabilization (MOMP) (38). Changes in MOMP and other mitochondrial dysfunctions may regulate the migration of cancer cells via reactive oxygen species $(39,40)$. To elucidate the direct involvement of Drp1 in hypoxia-induced migration, silencing Drp1 by siRNA was achieved in MDA-MB-231 cells (Fig. 3A). Similar to Mdivi-1, silencing Drp1 efficiently inhibited hypoxia-induced mitochondrial fission and migration in MDA-MB-231 cells (Fig. 3B-D). Taken together, these results indicate the different response of non-metastatic MCF-7 and metastatic MDA-MB-231 cells to hypoxia in migration are due to Drp1-dependent mitochondrial dynamics.

Cisplatin is widely used in the treatment of cancers (19). Similar to other chemotherapeutic agents, the application of cisplatin faces a stubborn problem, the resistance of cancer cells (20). Cisplatin resistance can be intrinsic or acquired. Intrinsic resistance originates from certain featured gene expression profile in cancer cells. Recently, some evidence implies the possible role of mitochondrial dynamics in cisplatin resistance $(22,23)$. Drp1-dependent mitochondrial fission improves piceatannol-induced cisplatin sensitivity in ovarian cancer (23). By contrast, OPA-1-mediated mitochondrial fusion is potentially responsible for the acquired cisplatin resistance in neuroblastoma B50 rat cells (22). In a previous (18) and our present studies, Drp1 expression in non-metastatic MCF-7 was lower than that in metastatic MDA-MB-231 cells. Furthermore, it has been reported that MCF-7 cells were more resistant to cisplatin than MDA-MB-231 cells (24). To investigate the role of intrinsic Drp1-dependent mitochondrial dynamics in cisplatin resistance, mitochondrial morphology after cisplatin was examined in two breast cancer cell lines. CDDP induced mitochondrial fission and significant apoptosis in MDA-MB-231, but not in MCF-7 cells (Fig. 4). Moreover, inhibition of Drp1-dependent mitochondrial fission by Mdivi-1 or silencing Drp1 efficiently attenuated CDDPinduced ROS production and MMP decrease (Figs. 6 and 7), and consequently alleviated the apoptosis of MDA-MB-231 cells induced by CDDP (Fig. 5). These results suggest that the cisplatin resistance in MCF-7 cells may originate from the weak Drp1-dependent mitochondrial fission. Instead, the strong Drp1-dependent mitochondrial fission facilitates the sensitivity of MDA-MB-231 cells to CDDP. Therefore, the intrinsic Drp1-dependent mitochondrial dynamics regulates the antineoplastic activity of cisplatin in breast cancer cells, although the underlying mechanism still needs further investigation.

In conclusion, our data show for the first time that Drp1-dependent mitochondrial fission regulates not only hypoxia-induced migration, but also the antineoplastic activity of cisplatin in breast cancer cells. Drp1-dependent mitochon- drial fission enhances the migratory activity in metastatic MDA-MB-231 cells under normoxia and hypoxia. In contrast, the lower Drp1 expression impedes the migration of nonmetastatic MCF-7 cells under normoxia and its response to hypoxia. On the other hand, Drp1-dependent mitochondrial fission sensitizes MDA-MB-231 cells to cisplatin. The weak Drp1-dependent mitochondrial fission leads to cisplatin resistance in MCF-7 cells. Taken together, Drp1-dependent mitochondrial fission not only promotes metastatic activity of breast cancer cells, but also facilitates its sensitivity to chemotherapeutic agents. Thus, our data suggest that Drp1 may be a potential target for suppressing breast cancer metastasis and improving its chemotherapeutic effect.

\section{Acknowledgements}

This study was supported by the National Natural Science Foundation of China (31360241 and 81472371), the Postgraduate Student Foundation for New Teacher from the Ministry of Education of China (20123601120001), Foundation from Department of Education of Jiangxi Province (GJJ13162) and Graduate innovative special fund project of Jiangxi province (YC2013-S001).

\section{References}

1. Jemal A, Bray F, Center MM, Ferlay J, Ward E and Forman D: Global cancer statistics. CA Cancer J Clin 61: 69-90, 2011.

2. Lu X, Yan $\mathrm{CH}$, Yuan M, Wei Y, Hu G and Kang Y: In vivo dynamics and distinct functions of hypoxia in primary tumor growth and organotropic metastasis of breast cancer. Cancer Res 70: 3905-3914, 2010.

3. Friedl P, Locker J, Sahai E and Segall JE: Classifying collective cancer cell invasion. Nat Cell Biol 14: 777-783, 2012.

4. Hanahan D and Weinberg RA: Hallmarks of cancer: the next generation. Cell 144: 646-674, 2011.

5. Nguyen DX, Bos PD and Massagué J: Metastasis: from dissemination to organ-specific colonization. Nat Rev Cancer 9: 274-284, 2009.

6. Lu X and Kang Y: Organotropism of breast cancer metastasis. J Mammary Gland Biol Neoplasia 12: 153-162, 2007.

7. Chan DA and Giaccia AJ: Hypoxia, gene expression, and metastasis. Cancer Metastasis Rev 26: 333-339, 2007.

8. Nagelkerke A, Bussink J, Mujcic H, Wouters BG, Lehmann S, Sweep FC and Span PN: Hypoxia stimulates migration of breast cancer cells via the PERK/ATF4/LAMP3-arm of the unfolded protein response. Breast Cancer Res 15: R2, 2013.

9. Krishnamachary B, Berg-Dixon S, Kelly B, et al: Regulation of colon carcinoma cell invasion by hypoxia-inducible factor 1 . Cancer Res 63: 1138-1143, 2003.

10. Muñoz-Nájar UM, Neurath KM, Vumbaca F and Claffey KP: Hypoxia stimulates breast carcinoma cell invasion through MT1-MMP and MMP-2 activation. Oncogene 25: 2379-2392, 2006.

11. Zhong H, De Marzo AM, Laughner E, et al: Overexpression of hypoxia-inducible factor lalpha in common human cancers and their metastases. Cancer Res 59: 5830-5835, 1999.

12. Oakes SA and Korsmeyer SJ: Untangling the web: mitochondrial fission and apoptosis. Dev Cell 7: 460-462, 2004.

13. Szabadkai G, Simoni AM, Chami M, Wieckowski MR, Youle RJ and Rizzuto R: Drp-1-dependent division of the mitochondrial network blocks intraorganellar $\mathrm{Ca}^{2+}$ waves and protects against $\mathrm{Ca}^{2+}$-mediated apoptosis. Mol Cell 16: 59-68, 2004.

14. Shaw JM and Nunnari J: Mitochondrial dynamics and division in budding yeast. Trends Cell Biol 12: 178-184, 2002.

15. Han XJ, Lu YF, Li SA, Tomizawa K, Takei K, Matsushita M and Matsui $\mathrm{H}$ : Involvement of calcineurin in glutamate-induced mitochondrial dynamics in neurons. Neurosci Res 60: 114-119, 2008.

16. Chan DC: Mitochondrial fusion and fission in mammals. Annu Rev Cell Dev Biol 22: 79-99, 2006. 
17. Grandemange S, Herzig S and Martinou JC: Mitochondrial dynamics and cancer. Semin Cancer Biol 19: 50-56, 2009.

18. Zhao J, Zhang J, Yu M, et al: Mitochondrial dynamics regulates migration and invasion of breast cancer cells. Oncogene 32: 4814-4824, 2013.

19. Wong E and Giandomenico CM: Current status of platinumbased antitumor drugs. Chem Rev 99: 2451-2466, 1999.

20. Milacic V, Fregona D and Dou QP: Gold complexes as prospective metal-based anticancer drugs. Histol Histopathol 23 101-108, 2008

21. Chen H, Hardy TM and Tollefsbol TO: Epigenomics of ovarian cancer and its chemoprevention. Front Genet 2: 67, 2011.

22. Santin G, Piccolini VM, Barni S, et al: Mitochondrial fusion: a mechanism of cisplatin-induced resistance in neuroblastoma cells? Neurotoxicology 34: 51-60, 2013.

23. Farrand L, Byun S, Kim JY, et al: Piceatannol enhances cisplatin sensitivity in ovarian cancer via modulation of $\mathrm{p} 53$, X-linked inhibitor of apoptosis protein (XIAP), and mitochondrial fission. J Biol Chem 288: 23740-23750, 2013.

24. Kurbacher CM, Wagner U, Kolster B, Andreotti PE, Krebs D and Bruckner HW: Ascorbic acid (vitamin C) improves the antineoplastic activity of doxorubicin, cisplatin, and paclitaxel in human breast carcinoma cells in vitro. Cancer Lett 103 : 183-189, 1996.

25. Wan YY, Zhang JF, Yang ZJ, et al: Involvement of Drp1 in hypoxia-induced migration of human glioblastoma U251 cells. Oncol Rep 32: 619-626, 2014.

26. Han XJ, Lu YF, Li SA, et al: CaM kinase I alpha-induced phosphorylation of Drp1 regulates mitochondrial morphology. J Cell Biol 182: 573-585, 2008.

27. Han XJ, Tomizawa K, Fujimura A, Ohmori I, Nishiki T, Matsushita $\mathrm{M}$ and Matsui $\mathrm{H}$ : Regulation of mitochondrial dynamics and neurodegenerative diseases. Acta Med Okayama 65: 1-10, 2011.

28. Park $\mathrm{J}$ and Choi $\mathrm{C}$ : Contribution of mitochondrial network dynamics to intracellular ROS signaling. Commun Integr Biol 5: 81-83, 2012.

29. Twig G and Shirihai OS: The interplay between mitochondrial dynamics and mitophagy. Antioxid Redox Signal 14: 1939-1951, 2011.
30. Zhang Y, Liu Q, Wang F, et al: Melatonin antagonizes hypoxiamediated glioblastoma cell migration and invasion via inhibition of HIF-1 $\alpha$. J Pineal Res 55: 121-130, 2013.

31. Chatterjee A, Dasgupta S and Sidransky D: Mitochondrial subversion in cancer. Cancer Prev Res (Phila) 4: 638-654, 2011

32. Galluzzi L, Morselli E, Kepp O, et al: Mitochondrial gateways to cancer. Mol Aspects Med 31: 1-20, 2010.

33. Ralph SJ, Rodríguez-Enríquez S, Neuzil J, Saavedra E and Moreno-Sánchez R: The causes of cancer revisited: 'mitochondrial malignancy' and ROS-induced oncogenic transformation - why mitochondria are targets for cancer therapy. Mol Aspects Med 31: 145-170, 2010.

34. Wallace DC: Mitochondria and cancer. Nat Rev Cancer 12: 685-698, 2012.

35. Rehman J, Zhang HJ, Toth PT, et al: Inhibition of mitochondrial fission prevents cell cycle progression in lung cancer. FASEB J 26: 2175-2186, 2012.

36. Hagenbuchner J, Kuznetsov AV, Obexer P and Ausserlechner MJ: BIRC5/Survivin enhances aerobic glycolysis and drug resistance by altered regulation of the mitochondrial fusion/fission machinery. Oncogene 32: 4748-4757, 2013.

37. Qian W, Wang J and Van Houten B: The role of dynamin-related protein 1 in cancer growth: a promising therapeutic target? Expert Opin Ther Targets 17: 997-1001, 2013.

38. Kushnareva Y, Andreyev AY, Kuwana T and Newmeyer DD Bax activation initiates the assembly of a multimeric catalyst that facilitates Bax pore formation in mitochondrial outer membranes. PLoS Biol 10: e1001394, 2012.

39. Ma J, Zhang Q, Chen S, et al: Mitochondrial dysfunction promotes breast cancer cell migration and invasion through HIF $1 \alpha$ accumulation via increased production of reactive oxygen species. PLoS One 8: e69485, 2013.

40. Tochhawng L, Deng S, Pervaiz S and Yap CT: Redox regulation of cancer cell migration and invasion. Mitochondrion 13: 246-253, 2013. 